\title{
Rechtsgeschichte
}

http://www.rg-rechtsgeschichte.de/rg8

$\operatorname{Rg} 2006 \quad 14-32$

Zitiervorschlag: Rechtsgeschichte Rg 8 (2006)

http://dx.doi.org/10.12946/rg08/014-032

\section{Marc Amstutz}

Vaios Karavas

\section{Rechtsmutation}

$\mathrm{Zu}$ Genese und Evolution des Rechts im transnationalen Raum 


\section{Abstract}

In order to cope with transnational law, we have to abandon hierarchical legal models which, up to the present, have dominated western legal discourse. In the emergence of a new world society, law is undergoing a mutation. This mutation is here understood as a new form of interaction with legal texts. While law has been interpreted until now with regard to auctoritas, i.e. to an external reference (e. g. God, the King, the Pope, the Legislator), this mode of interaction with the legal text can no longer grasp new normative phenomena which in the recent literature have been subsumed under the concept of transnational law. The authors take inspiration from the Jewish model of interpretation of legal texts - as an example of an alternative and more adequate approach to global legal phenomena - and try to elaborate this argument on the basis of European private law. 


\section{Rechtsmutation}

$\mathrm{Zu}$ Genese und Evolution des Rechts im transnationalen Raum

[I]l y avait du sacré dans l'accomplissement de ces ordres venus de tous et de nulle part.

Volodine (I985) 33

Die Metapher der legal transplants ${ }^{\mathbf{I}}$ weckt den Eindruck der Internationalität, der Grenzüberschreitung: Recht wandert nicht innerhalb eines Landes, sondern im großen Raum der Welt, also im Welt-Raum. Dieser Eindruck täuscht aber. Die Denkfigur des Rechtstransplantates entspringt der Vorstellungswelt des Nationalstaates. Ihre Verwendung blieb bislang auf nationales Recht beschränkt. Deshalb sollte einmal die Frage gewagt werden: Gibt es auch Normtransfers jenseits des Nationalstaates, also Transfers von nationalem Recht in den transnationalen Raum?

Im Spiegel der Lehre scheint es um die Chancen eines solchen Transfers schlecht bestellt zu sein. Der Möglichkeit von Recht im transnationalen Raum wird mit großer Skepsis begegnet, ja sogar mit Todesahnungen. Niklas Luhmann, der coole Beobachter, der distanzierte Soziologe, hat einst mit seinem typischen Vollstreckerstil gemeint, dass das Recht im Antlitz der Weltgesellschaft die Möglichkeit seines eigenen Endes einkalkulieren müsse: »Es kann ... durchaus sein, dass die gegenwärtige Prominenz des Rechtssystems ... nichts weiter ist als eine europäische Anomalie, die sich in der Evolution einer Weltgesellschaft abschwächen wird. « ${ }^{2}$ Mit einer solchen Hypothese ist er nicht allein: Pierre Legendre, der machtsensible Psychoanalytiker und Jurist, erblickt ganz ähnlich in der globalen Gesellschaft sich abzeichnende Tendenzen zur Abschaffung des Rechts - dieser zivilisatorischen
Errungenschaft, die den Menschen von einem bloßen Fleischkloß zum Menschen erhebt. ${ }^{3}$ Auch Rainer Maria Kiesow hat jüngst - kynisch-provokativ - einen Verfall des Rechts in der Weltgesellschaft behauptet: »Weltrecht Ruinenrecht $! \ll^{4}$ Nicht viel anders sieht es schließlich der strenge Analytiker Ralf Michaels: Für das um Befreiung von seinen territorialen Fesseln sich bemühende Recht prophezeit er zwar nicht den Tod, aber doch den Übergang in eine Lage gesellschaftlicher Anomie. Deshalb ist er mit sämtlichen argumentativen Mitteln bestrebt, seinen Patienten vor allen möglichen (mit dem Präfix »trans-« ausgestatteten) Viren zu schützen, indem er ihn künstlich im Zustand seiner nationalstaatlichen Verwurzelung behält. ${ }^{5}$

Auch wenn Interesse und Perspektiven dieser Autoren sehr unterschiedlich sind, haben sie doch dieselbe Frage im Visier: die Möglichkeit eines Rechts in der globalisierten Welt, eines Weltrechts also. Und alle sind sie sich darin einig: In der Weltgesellschaft sind die Aussichten für das Recht ziemlich düster. Aus welchem Grund aber sollte mit der Globalisierung das Totengeläut für das Recht erschallen? Wieso sollte dieses in der Atmosphäre des Transnationalen nicht überleben? Warum die brutal-simplizistische Gleichung »Weltgesellschaft = Rechtssterben «? Betrachtet man nochmals die angeführten Lehrmeinungen genau, so ist eines auffallend: Alle bauen sie stillschweigend auf einem identischen, sehr spezifischen Rechtsverständnis auf. Dieses Verständnis von Recht ist dasjenige der westlichen Rechtskultur - mit Legendre: das römischchristliche Rechtsmodell. ${ }^{6}$ Es führt alle Rechtsoperationen auf eine »oberste « Norm, auf Kel-

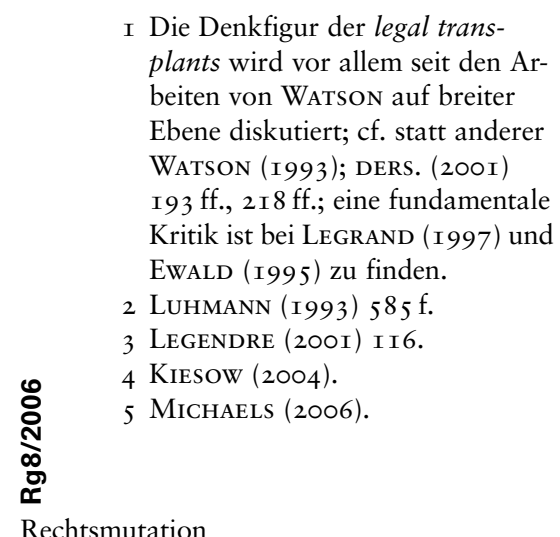

Die Denkfigur der legal transbeiten von WATSON auf breiter Ebene diskutiert; cf. statt anderer WATSON (I993); DERS. (200I) Iff., 2 I 8 ff.; eine fundamentale EWALD (I995) zu finden.

2 LuHManN (I993) $585 \mathrm{f}$.

(2004).

5 Michaels (2006). 
sens Grundnorm, ${ }^{7}$ zurück und baut damit eine hierarchische Normenpyramide. Dieses Modell gilt heute sowohl dem civil als auch dem common law als selbstverständlich. So selbstverständlich, dass seine Spezifizität schon gar nicht mehr ins Auge sticht.

Diese Spezifizität bildet nun aber, so meinen wir, den blinden Fleck der sowohl im kontinentaleuropäischen als auch im angelsächsischen Schrifttum geführten Diskussion über die Lebenschancen eines Weltrechts. Folgt man diesem Befund, wird erkennbar, weshalb Juristen, Psychoanalytiker und Soziologen gleichermaßen der Vision eines Schwundes des Rechts im transnationalen Raum das Wort reden: In diesem sind die gewohnten Rechtshierarchien - Hierarchie der Regeln, Hierarchie der Rechtsquellen, Hierarchie der Spruchkörper usw. - einfach inexistent. ${ }^{8}$ Der transnationale Raum ist ein Raum ohne Rechtsgeometrie! Was Wunder dann, dass das hierarchische Rechtsmodell zum Verständnis eines transnationalen Rechts nicht weiterhilft, dass im Lichte dieses Modells Recht in den anationalen Räumen der Weltgesellschaft an Plausibilität massiv einbüßt.

Und in der Tat: Dass unser westliches Rechtsbild, welches auf Kohärenzgebote wie (lobend-anerkennend) die »Einheit der Rechtsordnung « 9 oder (sarkastisch-karikierend) die »Tyrannei der Werte ${ }^{\text {Io }}$ setzt, in der pluralisierenden Globalisierung kaum Hoffnung auf Erfolg zulässt, sei den eingangs genannten Autoren getrost zugestanden. ${ }^{\text {II }}$ Aber ihre Überlegungen bauen auf eine nicht hinterfragte Prämisse auf, die sich als fatal erweist: Unterschwellig argumentieren sie allesamt mit dem eingangs angesprochenen Transfermuster, mit dem Szenario einer Transplantation des pyramidenförmig hierarchisierten Rechts des Nationalstaates in den transnationalen Raum. Dabei verlieren sie die
Eigenschaften und Idiosynkrasien des Transplantates aus den Augen und übersehen dessen fundamentale Inkompatibilität mit dem empfangenden »Körper «. Fast hat man den Eindruck, als verflüchtigte sich dieser Umstand in der dunklen Ästhetik der "grandiosity of law in the ruins $",{ }^{\mathrm{I} 2}$ deren morbider Faszination die zum Weltrecht sich äußernden Autoren zuweilen nur mit Mühe widerstehen.

Aber transnationales Recht ist nicht Ruinenrecht. Eine vielleicht auf den ersten Blick eher unscheinbar daher kommende, im Grunde aber folgenreiche inversio terminorum erlaubt, dies zu sehen: Genese und Evolution von Weltrecht mit Hilfe der Metapher eines Rechtstransfers von nationalem Recht in den transnationalen Raum zu analysieren, führt zu nichts, oder besser: zum Nichts, nämlich zu Nekrologien des Rechts. Was in Wahrheit stattfindet, ist etwas ganz anderes: ein Prozess, den wir nicht als einen solchen des Transfers, sondern als einen solchen der Mutation zu verstehen lernen müssen. Unsere These: Das hierarchische Rechtsmodell der westlichen Rechtskulturen durchläuft in der emergierenden Weltgesellschaft eine Mutation, die es zu »etwas anderem " macht. Das Recht mutiert - und dies bedeutet insbesondere: seine Normativität wird im transnationalen Raum umgeformt, sie wird »trans-formiert «. Damit erst wird erkennbar, wie verheerend sich der Verlust der Territorialität auf das herkömmliche Rechtsverständnis auswirkt: Alle uns vertrauten juristischen Legitimations-, Interpretations- und Begründungsmuster verlieren ihre Basis. ${ }^{{ }^{3} 3}$ Das mutierende Recht der Transnationalität löst sich allmählich von unserem Rechtsverständnis, auch wenn es, was im Einzelnen noch darzustellen sein wird, Recht ist und bleibt. Diese These entfalten wir in drei Schritten:

Zunächst analysieren wir die Züge des hierarchischen Rechtsmodells, und zwar mit einer

\footnotetext{
7 Kelsen (I960).

8 Cf. in diesem Zusammenhang

Vesting (2004); LADEUR (I999);

Teubner (I996a).

9 ENGISCH (1935).

iо Sснмiтt (I967).

I I Cf. in diesem Zusammenhang auch LADEUR (2004) 97.

I 2 Teubner (2003) 25 mit Verweisung auf KeNNEDY (I989) 5 I 6 .

I3 Cf. in diesem Zusammenhang etwa Berman (2002) 3I $8 \mathrm{f}$.
} 
doppelten Zielsetzung: Einerseits wollen wir die Ursachen seiner »organischen « Inkompatibilität mit dem transnationalen Raum ergründen, und andererseits die »Gene « im Körper - oder präziser: im »Genom « - dieses Modells identifizieren, die in der Transnationalisierung mutieren (I.)

Sodann gehen wir näher auf Studien über alternative Formen von Rechtsnormativität ein, die sich unter anderem vom jüdischen Rechtsmodell anregen lassen (II.). Dass gerade in diesem Modell Versatzstücke für die Konstruktion eines transnationalen Rechts vorhanden sein könnten, lässt schon die Geschichte des jüdischen Rechts vermuten: "The Jewish legal system has evolved for the past I900 years without a state and largely without much in the way of coercive powers to be exercised upon the ... [members of Jewish communities]. « ${ }^{\mathrm{I} 4}$

Schließlich werden wir die Einsichten, die diese experimentellen Studien gewonnen haben, im Kontext der Weltgesellschaft zu generalisieren versuchen. Auf diesem Weg sollen erste Konturen eines transnationalen Rechts gezeichnet werden, das diesen Namen auch wirklich verdient, d.h. Konturen eines Rechts, das sich in den Interstizien zwischen Nationalstaaten herausbildet und (in der Hauptsache) fernab von Staatlichkeit operiert. Immer gilt aber: verba docent, exempla trahunt. Deshalb soll abschließend das identifizierte (mutierte) Rechtsmodell, das wir in ersten Ansätzen im transnationalen Recht erkennen, an einem konkreten Gegenstand beobachtet werden: am Beispiel des Europäischen Privatrechts. Es wird sich zeigen, dass sich in der Praxis dieses Rechtsgebiets bereits bedeutsame Kerngedanken des von uns ausgemachten transnationalen Rechtsmodells verdichtet haben (III.).
I. Das Genom: Nationalstaat und

hierarchisches Rechtsmodell

Das hierarchische Rechtsmodell der westlichen Rechtskulturen ist nicht das Ergebnis eines Entwurfes, das ein Jurist, ein Politologe oder ein Staatswissenschaftler mit ratio und reflexio erarbeitet hätte. Vielmehr ist es das Produkt blinder Sozialevolution. Nicht taxis, sondern kosmos. ${ }^{{ }^{15}}$ Aber wie hat sich dieses Modell herausgebildet? Welche Umstände haben es begünstigt? Ein möglicher (wenn auch bei weitem nicht der einzig denkbare) Ansatzpunkt, um dies zu verfolgen, ist die Beobachtung der Umwälzungen des kontinentaleuropäischen Justizwesens im Verlauf des I8. und I9. Jahrhunderts.

Während dieser Zeitspanne emanzipieren sich die Gerichte allmählich vom politischen Willen, d. h. vom Fürsten, der sich seit der Neuzeit die Interpretation seiner Gesetze vorbehalten, diese also dem Richter verboten hatte. ${ }^{\mathbf{I}}$ Der Weg dieser Emanzipation war jedoch steinig. Erst gegen Mitte des I8. Jahrhunderts kam es zu vereinzelten Demarkationen der Einmischung des Fürsten in die Rechtsprechung, bis sich dann schließlich die Forderung bis zur Restauration durchsetzte. ${ }^{17}$ Mehr oder weniger parallel zu dieser Ausdifferenzierung findet in Europa eine spezifische Form der Herrschaftsbildung und Ethnogenese statt: der Übergang vom Staat zum Nationalstaat. ${ }^{18}$ Man kann diese Transformationen als funktionale Ausdifferenzierung von Recht und Politik und als weitere Schritte im Prozess der autopoietischen Schließung dieser Systeme sehen.

Für das Recht haben diese gesellschaftlichen Umbrüche und Reorganisationen eine kaum zu überschätzende Konsequenz: Diesem geht im Soge dieser Ausdifferenzierungs- und Schließungsprozesse der Zugang zu den Machtressour-

\footnotetext{
I4 COVER (I987) 68.

I 5 Hayek (I973) 37: »Classical

Greek was ... fortunate in possessing distinct single words for the two kinds of order, namely taxis for a made order ..., and kosmos for a grown order... i6 Cf. etwa D. Simon (I975) 2; ferner auch T. SiMON (2005). I7 D. Simon (I975) 3.

I 8 LANGEWIESCHE (2000) 28: »Trotz aller Vorstufen ist der Übergang

vom Staat zum Nationalstaat nicht vor dem späten $\mathrm{I} 8$. bzw. dem I9. Jahrhundert anzusetzen. « 
cen des Staates verloren. Der Nationalstaat konzentriert die Macht bei der Politik, was für das Recht das Problem der Durchsetzbarkeit seiner Anordnungen aufwirft: Wie kann das Recht das angeordnete Handeln oder Unterlassen erzwingen, wenn es über die dafür erforderliche Macht nicht unmittelbar verfügt? Seine Normativität wäre gefährdet, müsste es dauernd sagen: »Du hast zwar recht, aber leider können wir Dir nicht helfen. " ${ }^{\text {I9 }}$ Deshalb ist, wie Luhmann sagt, eine "gewisse Funktionssynthese von Politik und Recht « unerlässlich. ${ }^{20}$ Eine solche »Funktionssynthese « ist aber eine hochkomplexe und alles andere als ungefährliche Angelegenheit, weil damit die Möglichkeit eines Einbruchs der Politik in die Rechtswelt geschaffen wird. Diese Möglichkeit begründet die Gefahr, dass das proprium des Rechtssystems korrumpiert oder zerstört wird. Aus diesem Grund hat das Recht allen Anlass, ein Arrangement zu suchen, das die Autonomie seiner Operationen trotz der Angewiesenheit auf die Politik bewahrt. Wie gelingt das?

Die (Zauber-?) Formel heißt: das nationalstaatliche Gesetz. Seine Besonderheit liegt in seiner Doppelwertigkeit: Die Verabschiedung eines Gesetzes im Parlament ist ein rein politischer Akt. Aus der Sicht des Rechtssystems ist das Gesetz vorerst nichts anderes als eine Irritation, vergleichbar mit anderen Irritationen aus der Wirtschaft oder aus der Kunst. Die politische Tätigkeit der Gesetzgebung stellt für das Recht ein bloßes »Rauschen " dar, das dieses aber in Informationen transformieren kann. Denn das Gesetz ist ein Fall struktureller Kopplung von Recht und Politik. Das bedeutet, dass es für das Rechtssystem einen "Bereich « bildet, in dem »Möglichkeiten gespeichert [sind], die das System verwenden kann, die es in Informationen transformieren kann ", ${ }^{2 \mathrm{I}}$ aber nicht muss. Was aber macht das Recht mit dem Gesetz in con- creto? Beobachten wir zunächst, was das Rechtssystem mit Hilfe des Gesetzes nicht machen kann, und das ist: Machtressourcen aus der Politik in den eigenen Funktionsbereich "physisch « zu »importieren ", um Zwang selber auszuüben. Das verbietet ihm schon seine operative Geschlossenheit.

Dennoch spielt das Gesetz in diesem Zusammenhang eine entscheidende Rolle: Über das Gesetz - als Form der strukturellen Kopplung mit der Politik - verschafft sich das Recht die erforderlichen Informationen, die in der Rechtskommunikation als Symbol für jederzeitige Durchsetzbarkeit der getroffenen Anordnungen fungieren. In dieser Schaffung einer Symbolik der Rechtsdurchsetzung bzw. einer Geltungssymbolik liegt denn auch die erwähnte »Funktionssynthese « Recht/Politik. Darin liegt aber zugleich auch die Basis der Normativität des Rechts. Denn: Dass das Recht über die Mittel für seine eigene Durchsetzung nicht verfügt, wird durch die eben beschriebene Symbolisierung invisibilisiert. Dies insofern, als die Effektivität des Symbols davon herrührt, dass es unbemerkt für das Symbolisierte steht, d.h. dass »es nicht als Eigenständiges, nicht als Symbol erkannt bzw. wahrgenommen und empfunden wird, sondern im Wesentlichen als identisch mit dem Symbolisierten ${ }^{22}$

Aber diese Geltungssymbolik hat ihren Preis, den Preis einer phantastischen Fiktion, die durch die Erfindung des modernen Gesetzgebers vollzogen wird. Mit dieser Erfindung wird ein ganz bestimmter Umgang mit dem Gesetz d. h.: mit dem Text - festgelegt. Just dieser Umgang mit dem Text, der in den westlichen Rechtskulturen auf Dauer gestellt ist, bildet das »Gen «, das, so werden wir sehen, im transnationalen Raum mutiert. Um diese Zusammenhänge zu erläutern, müssen wir näher auf den erwähnten
I9 LUHMANN (I993) I 53.

20 LUHMANN (I993) I 53; DERS. (I98I) I $54 \mathrm{ff}$.

2 I LUHMANN (2002) I 2 I.

22 Niggli (2000) I40; mit der Autopoiese des Rechts ist die Symbolisierung von Rechtsgeltung durch einen politischen Akt, d. h. durch das Gesetz, durchaus vereinbar. Erkennbar wird das vor allem daran, dass es dem Recht über das Gesetz zugleich - also nebst dem Wettmachen seiner Durchsetzbarkeitsdefizite - gelingt, die Politik auf Distanz zu halten (und so sein proprium vor politischen »Immissionen « $\mathrm{zu}$ bewahren). Denn es bleibt frei, das Gesetz zu beachten oder nicht; cf. zum Ganzen eingehend AmstuTz (2003a). 
westrechtlichen "ganz bestimmten Umgang mit dem Text « eingehen, den Legendre als römischchristlichen charakterisiert. Dafür ist erforderlich, das I9. Jahrhundert zu verlassen und das Rad der Zeit bis zu den juristischen Autoren der Scholastik zurückzudrehen. Denn sie sind es, so Legendre, die vom römisch-christlichen Umgang mit dem Text der Legalität am meisten verstanden haben. Bei ihnen könne man lernen, was das wahre Gesicht dieses Umganges sei.

Nun: Wie gehen denn die Juristen der Scholastik mit ihren Texten um? Legendres Antwort verwirrt zunächst einmal: Diese Juristen hätten »mit der Nase an ihren Texten « geklebt. Die Verwirrung weicht aber schnell einer tief gehenden Einsicht. Denn Legendre präzisiert: "An ihren Texten klebend, ja, aber genau in der von der römisch-christlichen Legalität autorisierten Art und Weise. "Den Scholastikern komme es nämlich nicht auf den Text als solchen an. Was in ihren Augen zähle, sei »die ratio des Textes, buchstäblich die Vernunft $\ll .{ }^{23}$ Und im Anschluss an diesen Befund hält Legendre fest: »Die Vernunft ist im Text enthalten, und das ist, so scheint mir, ein kapitaler Punkt. " ${ }^{24}$

Weshalb scheint Legendre dieser Punkt »kapital «? Weil, so seine Antwort, ein Text in der scholastischen Ordnung der Schrift vor allem auctoritas ist. Auctoritas bewirkt im Ritual des Rechts, dass der Text "einen bestimmten liturgischen Platz einnimmt «. Er dient dann als "gesetzesmäßige Referenz ..., in der Weise, dass eine auctoritas immer die Wahrheit spricht; was immer sie sagt, man muss sich darauf einstellen «. ${ }^{25}$ Beruft man sich auf den Text, so beruft man sich auf auctoritas, auf einen »obersten gesetzeskräftigen Signifikanten ..., das heißt, was das Abendland des Mittelalters und die Scholastik anlangt, auf den Kaiser oder den Papst, auf eine Instanz, die als >lebende Stimme des Rechts (viva vox iuris), als >beseeltes Gesetz (lex animata) im Gesetz selbst genannt ist ${ }^{26}{ }^{26}$

Diese Möglichkeit eines Sich-Berufens auf auctoritas wurde mit der Erfindung des modernen Gesetzgebers am Leben erhalten: Nachdem im Nationalstaat die legalistische Autorität des Kaisers oder des Papstes geschwunden war, stand die Stelle, die letztere so lange besetzt hatten, leer. In die Bresche gesprungen ist der »nationalstaatliche" Gesetzgeber, dieses selbstreferentielle Phantom, das weder im Parlament, noch im einzelnen Parlamentarier, noch im parlamentarischen Diskurs, noch im Volk (als »Souverän «), noch in der Regierung seine Inkarnation gefunden hat. Heute noch beherrscht der Gesetzgeber bzw. sein Wille die kontinentaleuropäische Methodenlehre, d. h. das Korpus von Regeln der Anwendung von Gesetzen (von »Texten «), und dies mit dem einzigen Ziel, die Vorstellung, dass die Vernunft im Text enthalten ist, fortzusetzen. Jeder Richter, jeder Verwaltungsjurist, jeder Anwalt beruft sich auf die auctoritas "im " Text, und das bedeutet: Er beruft sich "[a]uf den Platz, den der Text in der Hierarchie der Texte, in der geweihten Reihung der Texte einnimmt $« .{ }^{27}$ Diese "geweihte Reihung der Texte" ist die uns zugleich so teure und so lästige Normpyramide Kelsens. Und ein anderer Name für "Gesetzgeber « ist - ganz scholastisch - ratio legis.

Jetzt dürfte auch klar werden: Ein solcher römisch-christlicher Umgang mit dem Text ist im transnationalen Rechtsraum ein Ding der Unmöglichkeit. Führen wir uns einmal konkrete Beispiele vor Augen, z. B. die lex mercatoria, ${ }^{28}$ die lex digitalis, ${ }^{29}$ die lex sportiva internationalis $^{3 \circ}$ oder "Regeln " der Corporate Governance, ${ }^{3 \mathrm{I}}$ die von Land $\mathrm{zu}$ Land umherziehen, ohne dass jemand sagen könnte, von wem sie denn »erlassen « wurden. Wer, welche Instanz ist die viva vox iuris, die "lebende Stimme" dieser

\footnotetext{
23 LEGENDRE (I989) 32.

24 LEGENDRE (I989) 32.

25 LEGENDRE (I989) 32.

26 LEGENDRE (I989) 33.

27 LEGENDRE (I989) 32.

28 Zur lex mercatoria statt anderer

Stein (I995); Mertens (I997);

Teubner (I998); Zumbansen

(2003).

29 Zur lex digitalis statt anderer

Post (i995); Calliess (200I);

Teubner, Karavas (2003).

30 Zur lex sportiva internationalis statt anderer NAFZiger (I996).

3I $\mathrm{Zu}$ den Corporate Governance Codes statt anderer Zumbansen (2002). 
leges? Auf welche auctoritas nimmt man Bezug, wenn man sich auf deren Texte beruft? Werden diese Texte von irgendeiner "Stimme« belebt, sind sie leges animatae? Die schroffe Antwort lautet: Nein. Kurz und bündig. Ohne Wenn und Aber. Und diese Antwort bedarf nicht einmal der Begründung, so selbsterklärend ist sie.

Man kann das mit dem Fehlen eines politischen Systems in der Weltgesellschaft in Verbindung bringen: Hier gibt es keine Souveränität, keine Entscheidungshierarchie, keine demokratische Willensbildung. ${ }^{32}$ Auch hilft nicht weiter, nach Surrogaten für eine » Weltpolitik « oder eine global public sphere zu suchen. ${ }^{33}$ Weder die Zivilgesellschaft, noch NGOs, noch multinationale Unternehmen können die Texte des transnationalen Rechts mit einer Stimme (welcher?) beseelen. ${ }^{34}$ Es scheint, als bleibe dem Recht nur ein einziger Ausweg: auf einen anderen Umgang mit dem Text als dem römisch-christlichen umzuschwenken. Der Umgang mit dem Text ist das »Gen « im corpus iuris westlicher Prägung, auf das wir fokussieren müssen. Aber wie muss man sich die Mutation dieses »Gens« vorstellen?

\section{Das Gen: Auctoritas und Text der Legalität}

Versteht man Recht als die Lehre eines besonderen Umganges mit dem Text, so fragt sich sofort: Wie kann mit einem Text anders als »römisch-christlich « umgegangen werden? Damit wird keineswegs auf die Kontroverse zwischen Positivismus und Dezisionismus angespielt, d.h. auf die (die rechtsmethodische Diskussion im vergangenen Jahrhundert dominierende) Alternative, entweder »das Urteil als Erkenntnis ... [zu stilisieren], dessen Inhalt aus den Rechtsquellen abzuleiten ist ", oder "als Willensakt zu konzipieren, dessen Inhaltsbestim- mung [durch den Richter] normativ einer creatio ex nibilo entspricht ". ${ }^{35}$ Die Frage ist vielmehr, ob jenseits dieser Alternative ein Rechtsmodell denkbar ist. Ein Rechtsmodell, das weder auf der ratio des Gesetzes noch (was ideengeschichtlich das gleiche ist) auf dessen kategorischer Ablehnung, also auf der ratio des Urteils (d.h. auf dezisionistischer Macht), noch auf einem Kompromiss zwischen Positivismus und Dezisionismus ${ }^{36}$ gründet. In der Auseinandersetzung mit dieser Frage könnte die Rechtstheorie viel von einer Kontroverse in der Texthermeneutik der letzten Jahrzehnte lernen, die heute vor allem mit dem Namen von Roland Barthes assoziiert wird.

Seit dem I9. Jahrhundert herrschte in der Literaturwissenschaft die Tendenz vor, Texte vornehmlich im Zusammenhang mit der Biografie des Autors und entsprechend psychologisierend zu deuten. Der Autor ist dieser herkömmlichen Auffassung nach (vor allem in der Nachfolge des romantischen Genie-Gedankens) nicht nur der Urheber eines Textes, sondern auch die Autorität, die seine Bedeutung bestimmte. Diesen originären Bedeutungs- oder Sinngehalt gilt es dementsprechend zu rekonstruieren, wenn nötig auch über autorbiografische Anhaltspunkte. Im Schrifttum des 20. Jahrhunderts finden sich indes immer wieder vereinzelte Ansätze, die einer auf die Autorität des Autors orientierten Texthermeneutik gegenüber kritisch eingestellt sind, so z. B. in der Poetik der Moderne, im angloamerikanischen New Criticism, ${ }^{37}$ in der Semiotik Umberto $\operatorname{Ecos}^{38}$ oder im Werk Michel Foucaults. ${ }^{39}$ Es ist jedoch Roland Barthes, der große Semiologe und Literaturkritiker, der den Tod des Autors in einem gleichnamigen Aufsatz aus dem Jahre 1968 in aller Radikalität verkündet hat. ${ }^{4 \circ}$

Der herkömmlichen autororientierten Lesart setzte Barthes eine rein textgestützte Interpretation entgegen und erhöhte so den Leser und seine
32 Cf. z. B. Teubner (I996a); CalLIESS (2002) 2 I 5.

33 Cf. Habermas (I998); HöfFe (I999).

34 Cf. in diesem Zusammenhang etwa NiCKel (2005) m. Nw. (mit Blick auf die Zivilgesellschaft und die NGOs); Joerges, NeYer (I997) (mit Blick auf die Europäische Gemeinschaft).

35 Fischer-Lescano, Christensen (2005) 2I5.
36 So die berühmte Soraya-Formel: BVerfGE 34, 269 ff., 293; dazu statt vieler RAFI (2004) 4I ff.

37 Es handelt sich um eine vor allem in den USA zwischen I920 und I970 aktive literaturkritische und -theoretische Bewegung; als Hauptvertreter dieser Richtung gelten: John Crowe Ransom, Allen Tate und Cleanth Brooks.

38 ECO (I998).

39 Foucault (2000).
40 BARTHES (2000). 
Deutungsfähigkeiten zur sinnstiftenden Instanz im Bedeutungsprozess. Damit wies er die traditionelle Theorie des Autors als sinnstiftende Instanz zurück und besetzte dessen Stelle mit dem Skriptor, einer Autor-Funktion, die erst in der Lektüre und damit im Text selbst zustande kommt. Allerdings ist der Skriptor nicht als transzendentaler Garant von Sinn zu denken, sondern als eine aus heterogenen Sprachspielen, diskursiven Anspielungen und kulturellen Praktiken zusammengesetzte rhetorische Funktion. Diese Umpolung von einer autororientierten hin zu einer leserorientierten Perspektive zeitigt nun gewichtige Implikationen für den Begriff des Textes selbst. Der Text wird nicht mehr als ein fertiges Produkt mit einer festen, zwar verborgenen, aber dennoch endgültig und für immer inskribierten Bedeutung betrachtet, sondern als eine offene Textur, deren Sinn durch den Leser stets neu aktualisiert wird. In Barthes' eigenen Worten: » Text heißt Gewebe; aber während man bisher dieses Gewebe als ein Produkt, einen fertigen Schleier aufgefasst hat, hinter dem sich, mehr oder weniger verborgen, der Sinn (die Wahrheit) aufhält, betonen wir jetzt bei dem Gewebe die generative Vorstellung, dass der Text durch ein ständiges Flechten entsteht und sich selbst bearbeitet; in diesem Gewebe - dieser Textur - verloren, löst sich das Subjekt auf wie eine Spinne, die selbst in die konstruktiven Sekretionen ihres Netzes aufginge. ${ }^{4 I}$

Betrachtet man all das aus juristischer Sicht, stellt sich die Frage: Kann man Barthes' Textbegriff auch im transnationalen Recht, wo es, wie gesagt, keinen »Autor-Gesetzgeber « gibt, fruchtbar machen? Sollte man versuchen, diesen Textbegriff in Zusammenhang zu bringen mit den vielfältigen und verschiedenartigen leges, die im »Welt-Raum « generiert werden und die jenseits von territorial eingegrenzten Staaten evo- luieren? Kann man den »Gesetzgeber « nationalstaatlicher Prägung verabschieden und durch einen »juridischen Skriptor « ersetzen? Was müsste man sich dann unter diesem "Skriptor " konkret vorstellen? Vielleicht sind diese Fragen aber immer noch allzu stark vom Bild des hierarchischen Rechtsmodells geprägt. Vielleicht müssen wir unsere westlichen Juristen-Reflexe noch radikaler unterdrücken. Denn es geht nicht darum, einen »Ersatz« für den Gesetzgeber zu finden; was auf dem Spiel steht, ist nichts weniger als eine andere Organisation der Normativität bzw., was dasselbe meint, ein alternativer Umgang mit dem Text der Legalität.

Dass die Einsätze, um die hier gespielt wird, nicht irgendwelche rechtstheoretischen Nuancen, sondern förmlich einen clash of cultures darstellen, hat Legendre am Beispiel der Justinianischen Novelle I46 aufgedeckt. Seine Aufmerksamkeit gilt einem Auszug daraus, nämlich der Formulierung: "... hebraei ... insensatis semetipsos interpretationibus tradentes ... " (»Die Juden interpretieren verrückt «), ${ }^{42}$ und die Frage ist, weshalb er gerade auf diesen Satz fokussiert. Seine verblüffende Antwort: Weil man daraus »über die Logik der abendländischen Institutionen; über den Stoff, aus dem die Institutionen ihre Kraft beziehen ", Entscheidendes erfährt. ${ }^{43}$ Was also auf den ersten Blick als eine bloß pejorative Beurteilung der Art und Weise erscheint, wie die Juden mit dem Gesetzestext umgehen, ist für Legendre von geradezu fundamentaler Bedeutung: Damit könne nichts Minderes als die Grundlagen der abendländischen menschlichen Existenz ergründet werden. ${ }^{44} \mathrm{Wie}$ ist das zu verstehen?

In der Zeit, als die Novelle I46 erlassen wurde, war die jüdische Religionsgemeinschaft von einer tiefen Kontroverse über die lokalen Idiome und ihren Gebrauch als Liturgiesprache

\footnotetext{
4I BARTHES (I986) 94.

42 Wie Legendre bemerkt, findet

sich die lateinische Fassung in

allen Ausgaben des Corpus Juris.

Der wiedergegebene Auszug ist

dem Prolog entnommen; cf. LE-

GENDRE (I989) Anm. I m. Nw.

43 LEgENDRE (I989) 22.

44 Näheres dazu bei LEgENDRE

(2004).
} 
erschüttert. Legendre hebt hervor, dass Justinians Novelle in dieser Kontroverse zugunsten der »laxistischeren Fraktion des Judentums « Partei ergreift. ${ }^{45}$ Aber in dieser historischen Kontingenz erschöpft sich für ihn ihre Bedeutung bei weitem nicht. Auch das kaiserliche Verbot der Mischna und ihres Kommentars, der Gemara, das in der Novelle I46 ausgesprochen wird, ist nicht das eigentlich Brisante. ${ }^{46}$ Und nebensächlich ist für Legendre schließlich, dass durch den Erlass dieser Novelle eine nicht mehr überwindbare Freund-Feind Opposition markiert wird, die man als Geburtsstunde des Antisemitismus ansehen könnte. ${ }^{47}$

Das wirklich Entscheidende an der Novelle I46 lokalisiert Legendre im Umstand, dass der Kaiser Justinian damit einen institutionellen Mechanismus erfunden hat, der seither im »industriellen Gesellschaftssystem « ungebrochen wirkt. Dieser Mechanismus besteht darin, durch die Instrumentalisierung des Signifikanten »Jude « den römisch-christlichen Zugang zum Gesetzestext als den einzig richtigen $\mathrm{zu}$ fixieren. Die Juden werden konkret als »Kategorie des christlichen Rechts « erfasst und sie werden dadurch zum Zweck der Zelebrierung der imperialen und theokratischen Wahrheit ausgenutzt. »Denn «, so Legendre weiter, »die Juden haben ihren Platz in der christlichen Legalität, und sie haben ihn als die Juden. Die Juden sind als solche eine Kategorie des christlichen Rechts; sie verkörpern das Von-Sinnen-Sein, sie steuern, von ihrem spezifischen Platz innerhalb des römisch-christlichen Legalismus aus - und das heißt eben: als Verrückte - das ihre zum Glanz der imperialen und theokratischen Wahrheit bei. ${ }^{4}{ }^{8}$

Was also Legendre behauptet, ist im Grunde genommen, dass der institutionelle Mechanis- mus der Novelle I46 nach dem Muster der ausschließenden Einschließung funktioniert: Indem die Juden als » verrückt « bezeichnet und aus dem System des imperialen-christlichen Legalismus ausgeschlossen werden, werden sie genau durch diese Ausschließung in das System wieder eingeschlossen. Und dies zum Zweck der Etablierung, d.h. der Institutionalisierung des Systems. Der Signifikant "Jude « sorgt m. a.W. für die Kohärenz des Systems; durch den Signifikanten »Jude « wird die Wahrheit des Systems zelebriert. ${ }^{49}$

Warum aber behauptet die Novelle I46, die Juden interpretierten »verrückt «? Was wird ihnen mit diesem Satz zur Last gelegt? Legendre meint, es sei zweierlei: Einerseits, dass sie Sklaven des Buchstabens sind, und andererseits, dass sie den Text bis zur Kastration interpretieren. ${ }^{\circ}$ Das sind natürlich keine leicht verständlichen Metaphern. Um den Vorwurf, die Juden seien Sklaven des Buchstabens, zu enträtseln, muss man mit Legendre in Betracht ziehen, dass der jüdische Zugang zum Text durch die Gemeinschaft der Interpreten vermittelt wird. ${ }^{5 \mathrm{I}} \mathrm{Im}$ Gegensatz zum römisch-christlichen Interpretationsmodell, das auf die Wahrsprüche einer "fleischgewordenen Macht « gegründet ist, ${ }^{52}$ fühlen sich die Juden bei ihrer Interpretation nicht durch eine außerhalb des Textes existierende ratio gebunden. Ihre Interpretation dient m.a.W. nicht der Zelebrierung einer außerhalb des Textes bestehenden Wahrheit, wie sie durch den Kaiser bzw. den Papst verkörpert wird. Im jüdischen Rechtsmodell existiert der Text nur auf Erden (nicht im Himmel). Eben deshalb erachten die christlichen Autoren der Antike den jüdischen Umgang mit dem Text als verrückt: Dieser Umgang kennt keine extern auferlegten Interpretationsgebote bzw. -grenzen. ${ }^{53}$
45 LEGENDRE (I989) 27.

46 LEGENDRE (I989) 27.

47 Wie LEgENDRE an anderer Stelle aufgezeigt hat, wäre eine solche Erklärung des Antisemitismus nicht nur oberflächlich, sondern sogar gefährlich; cf. LEGENDRE (200I).

48 LEgENDRE (I989) $28 \mathrm{f}$.

49 Der so verstandene Signifikant "Jude « weist Ähnlichkeiten auf mit Agambens homo sacer (cf. Agamben [2002]). Homo sacer war im archaischen römischen
Recht ein Mensch, den man straflos töten konnte, da er aus der Gemeinschaft verbannt und auf den Status seiner physischen Existenz reduziert war. Agamben benutzt nun diese obskure Figur als ein heuristisches Mittel, um gängige Gründungsmythen der souveränen Gewalt zu kritisieren. In seinen Augen wird die souveräne Gewalt nicht durch einen Sozialvertrag in welcher Form immer gegründet, sondern durch die ausschließende Einschließung des nackten Lebens in den Staat. In diesem Sinn ist der homo sacer nach Agamben die erste und unmittelbare Referenz der souveränen Macht.

5 O LEGENDRE (I989) 33.

5 I LEGENDRE (I989) 28.

52 LEGENDRE (I989) 28.

53 Cf. Legendre (I989) 33 f.: »Da die Juden weder einen Kaiser noch einen Papst haben, der ihnen die Wahrheit garantiert, stellt sich bei ihnen die Frage nach dem Signifikanten, mit der ein Subjekt ein 
Was hat es alsdann mit dem zweiten Vorwurf auf sich, die Juden würden den Text bis zur Kastration interpretieren? Da das Spiel mit dem Signifikanten bei den Juden viel radikaler und autonomer ausgestaltet ist, kann dieses Spiel nicht auf die Zementierung eines einzig richtigen Sinns beschränkt werden. ${ }^{54}$ Die Juden befreien den Text von jeglicher ratio - oder eben: Sie kastrieren ihn - und schaffen so einen Freiraum für die Sinnproduktion durch die Interpreten. Legendre weist in diesem Zusammenhang darauf hin, dass Kastration in der römisch-christlichen Tradition mit derselben Strenge geahndet wird wie Mord. ${ }^{55}$ Entsprechend schwerwiegend ist der Vorwurf, dass die Juden den Text interpretatorisch kastrieren. Er bedeutet nämlich nichts anderes, als dass sie durch Elimination jeglicher ratio aus dem Text unmittelbar die Wahrheitssprüche der fleischgewordenen Macht des Kaisers oder Papstes in Frage stellen. Oder metaphorisch: Durch den jüdischen Zugang zum Text wird der Kaiser bzw. der Papst selbst ermordet.

An dieser Stelle fallen die Parallelen zwischen der von Legendre beschriebenen jüdischen Interpretation und den Ausführungen von Barthes zum Tod des Autors ${ }^{56}$ auf. Wie erwähnt, stellt Barthes einen engen Zusammenhang zwischen der Produktion und der Rezeption literarischer Texte her. Er leugnet zwar nicht, dass literarische Texte immer einen Autor haben, aber, indem er den Text zu einem »Gewebe von Zitaten « erklärt, bringt er die Autonomie der künstlerischen Kreativität nahezu zum Verschwinden. Der Autor wird dadurch zum kompilatorischen Skriptor vorgegebenen Sprachmaterials reduziert. Deshalb fordert Barthes eine neue Art der Rezeption von literarischen Texten. Texte sollen nicht hermeneutisch im Hinblick auf einen richtigen und endgültigen Sinn entziffert werden; stattdessen sind ihre diffusen Sinngebungsstrategien zu ent- wirren. Diese neue Art der Rezeption literarischer Texte, die Barthes écriture nennt, ist insofern einschneidend, als sie - wie die jüdische Interpretation des Textes - eine Fixierung des Sinns radikal verweigert und dadurch die Wahrheitssprüche einer vorgegebenen Machtinstanz (im Fall von literarischen Texten: des Autors) abweist. In Barthes' Worten: »Die Schrift bildet Sinn, aber nur, um ihn wieder aufzulösen. Sie führt $\mathrm{zu}$ einer systematischen Befreiung vom Sinn. Genau dadurch setzt die Literatur (man sollte von nun an besser sagen: die Schrift), die dem Text (und der Welt als Text) ein >Geheimnis‘, das heißt einen endgültigen Sinn, verweigert, eine Tätigkeit frei, die man gegentheologisch und wahrhaft revolutionär nennen könnte. Denn eine Fixierung des Sinns zu verweigern, heißt letztlich, Gott und seine Hypostasen (die Vernunft, die Wissenschaft, das Gesetz) abzuweisen. « 57

Wenn man also die beiden Rechtsmodelle, das römisch-christliche und das jüdische, vor Augen führt, wird klar, dass wir es - wie schon erwähnt - mit einem clash of cultures zu tun haben. Denn das Verhältnis zwischen diesen beiden Arten des Zuganges zum Text kann nicht als ein dialektisches beschrieben werden. ${ }^{58}$ Wie Legendre betont, gibt es keine Hoffnung auf »Synthese «, »Versöhnung «, »Diskussion ", »Meinungsaustausch "zwischen den beiden Modellen in irgendeiner Zukunft. ${ }^{59}$ Im Gegenteil: Auseinandersetzung mit allen kriegerischen Mitteln ist die Losung. ${ }^{60}$

Während der jüdische Zugang zum Text bis heute nur die von Legendre beschriebene marginale Stellung im Rahmen des römisch-christlichen Modells einnahm, die ihm dieses zum Zweck der Untermauerung seiner eigenen gloria einräumte, könnte dieser Zugang heute im Soge der emergierenden Weltgesellschaft dramatisch an Aktualität gewinnen. Luhmann definiert die bestimmtes Spiel zu seiner Lust spielt, insofern grundlegend anders, als sie in einem Text enthalten ist. Im System der jüdischen Interpretationen ist das Spiel des Signifikanten als solchen autonomer, radikaler «.

54 LEGENDRE spricht an dieser Stelle von einer "Austrocknung aller Sinne durch die einheitliche Inter-

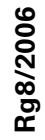
pretation eines kaiserlichen oder päpstlichen Rechts « (LEGENDRE [r989] 34).

55 Dazu näher LegendRe (I989) $36 \mathrm{ff}$.

56 BARTHES (2000).

57 BARTHES (2000) I9I.

58 So auch Legendre (I989) 28.

59 LEGENDRE (I989) 28.

60 LEGENDRE charakterisiert den römisch-christlichen Umgang mit dem Signifikant "Jude " als »Selbstverteidigung mit kriege- rischen Mitteln « (LEGENDRE [I989] 28). 
"Weltgesellschaft « als das umfassendste soziale System. ${ }^{61}$ Darunter versteht er eine emergente Ebene der gesellschaftlichen Ordnung, die sich nicht mehr in Zusammenhang zu nationalstaatlichen Denkkategorien bringen lässt. ${ }^{62}$ Diese emergente Ebene ist ein "Sinnhorizont" aller lokalen sozialen Ereignisse, der jenseits des $\mathrm{Na}$ tionalstaatlichen angesiedelt ist, ohne dass damit allerdings die globale Homogenisierung der Lebensbedingungen impliziert wäre. ${ }^{63}$ Insofern unterscheidet sich der Luhmannsche Gebrauch des Begriffes »Weltgesellschaft " von Konzepten, die die Globalisierung als Vernetzungsprozess zwischen nationalstaatlich geprägten gesellschaftlichen Ordnungen verstehen. ${ }^{64}$ Die »Weltgesellschaft « zeichnet sich in den Augen Luhmanns dadurch aus, dass sie keine sich selbst und ihre Teile beherrschende Ordnungsebene darstellt. Sie kann folglich nicht als eine politisch organi-

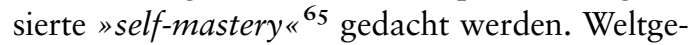
sellschaft und Weltstaat sind, folgt man dieser Sicht, zwei miteinander unvereinbare Konzepte.

Wie Anton Schütz bemerkt hat, ist das einzige Ordnungskonzept, durch welches sich die Weltgesellschaft beschreiben lässt, das Konzept der »order from noise ", d. h. das Konzept einer prekären, evolutorischen, kontigenten und unkontrollierten Ordnung. ${ }^{66}$ In einer so verstandenen Weltgesellschaft ohne Zentrum der Macht, ohne Hierarchien, ohne Normpyramiden, fehlen all jene Voraussetzungen, die für das Funktionieren des römisch-christlichen Rechtsmodells unerlässlich sind. Daraus geht hervor: Transnatio- nales Recht hat gar keinen anderen Ausweg, als mit dem römisch-christlichen Rechtsmodell zu brechen. Es wird aus strukturellen Gründen genötigt, »verrückt zu interpretieren «. Die Frage ist allerdings: In welche Richtung vollzieht sich dieser Bruch mit der westlichen Rechtskultur? Wie kann eine solche Befreiung von ratio, ein solcher Enthierarchisierungsprozess vor sich gehen? Eine Option, die das transnationale Recht so unsere These - im Begriff ist zu nutzen, ist die Mutation zu einem »entterritorialisierten "System, das Züge des jüdischen Rechtsmodells in sich aufnimmt. Das setzt aber voraus, dass es möglich ist, dieses Modell von seinen religiösen Bezügen zu lösen, es zu verweltlichen. Ist eine solche par force-Leistung denkbar?

Genau das bejaht Robert M. Cover in Studien, die unter Berufung auf die jüdische Rechtstradition, namentlich auf das Werk von Rabbi Joseph Caro, einem Mystiker aus dem I6. Jahrhundert, ${ }^{67}$ die These aufstellen, dass Recht keinen (National-)Staat voraussetzt. ${ }^{68}$ Covers Kernvorstellung besteht darin, dass von » Recht « nur die Rede sein kann, wenn dieses im »Universum « einer die Texte der Legalität interpretierenden Gemeinschaft (»nomic « oder »interpretative community«) entsteht. Dieses »Universum « nennt Cover Nomos. ${ }^{69}$ Darin wird Recht aus den interpretative commitments generiert, die in der fraglichen nomischen Gemeinschaft eingegangen werden, also aus den kollektiven Überzeugungen geschaffen, die die Weltanschauung dieser Gemeinschaft bestimmen und in dieser
6I LUHMANN (I97I).

62 So auch Burton (I972) 20.

63 Zum Konzept der »Weltgesellschaft « außerdem STICHWEH (200I); DERS. (2004); ferner auch Wobbe (2000).

64 Cf. dazu vor allem BeCK (I 997) und GidDens (I995).

65 SCHÜTZ (I997) 282: "... is quite obvious that world society does not imply planetary, politically organized self-mastery and that world society is not world-state."

66 SCHÜTZ (I997).

67 Cf. insbesondere Cover (I983) I 2 f.; zu Joseph Caro allgemein WERBLOWSKY (I962).

68 Cf. insbesondere Cover (I983); DERs. (I987). Diese These ist in der jüngeren Forschung in den verschiedensten Ausprägungen anzutreffen; cf. vor allem die Beiträge im Sammelband, der von Teubner (I997) herausgegeben wurde.

69 Cover (I983) 4: "We inhabit a nomos - a normative universe. We constantly create and maintain a world of right and wrong, of lawful and unlawful, of valid and void «; ferner op. cit. 7: »Law may be viewed as a system of tension or a bridge linking a concept of a reality to an imagined alternative that is, as a connective between two states of affairs, both of which can be represented in their normative significance only through the device of narrative. Thus, one constitutive element of a nomos is the phenomenon George Steiner has labeled >alternaty $`$ : the 'other than the case', the counter-factual propositions, images, shapes of will and evasion with which we charge our mental being and by means of which we build the changing, largely fictive milieu for our somatic and our social existence $<. .$. But the concept of nomos is not exhausted by its salternaty<; it is neither utopia nor pure vision. A nomos, as a world of law, entails the application of human will to an extant state of affairs as well as toward our visions of alternative futures. A nomos is a present world constituted by a system of tension between reality and vision." 
auch tatsächlich praktiziert werden. ${ }^{70}$ Interpretative commitments kreieren legal meaning und gerade dieser »Rechtssinn « ist für das Recht konstitutiv. Jede Interpretation des Texts der Legalität, die den interpretative commitments einer nomischen Gemeinschaft entspricht, hat folglich denselben Anspruch auf das Label »Recht«. Denn: Der Nomos braucht keinen Staat (auch wenn jeder Staat eines Nomos bedarf). Dementsprechend ist Recht - als Produkt einer nomischen Gemeinschaft - ein essentiell kulturelles Phänomen. ${ }^{71}$ Die Staatsgewalt, die durch eine spezifische Anwendung (Interpretation) von Recht ihren eigenen Nomos gegen den Nomos von Minderheiten im Staatsgebiet (z. B. von Feministinnen, Gewerkschaften, religiösen Gruppen, Green Peace, Tierschutzvereinigungen usw.) durchsetzt, ist in den Augen Covers problematisch, weil der Staat keinen ausschließlichen Anspruch auf das Attribut "Recht « hat. Der Nomos einer Minderheit, der sich vom "offiziellen « Nomos unterscheidet, ist nicht weniger Recht als das Recht des Staates. Ein Gericht, das sein Rechtsverständnis gegen dasjenige einer Minderheit durchsetzt, handelt jurispathisch. ${ }^{72}$

Wie ist mit Jurispathie umzugehen? Cover sucht einer Antwort dadurch näher zu kommen, dass er die Hauptprämisse des hierarchischen Rechtsmodells auf den Kopf stellt: Nicht Autorität verleihe die Befugnis zur Interpretation, sondern erst »Rechtssinn ", der interpretativ geschaffen werde, vermöge Autorität zu gründen. ${ }^{73}$ Das staatszentrierte, hierarchische Rechtsverständnis sei das Produkt einer Kategorien-Konfusion: Interpretation von Recht stelle keineswegs das Janusgesicht der politischen Gewalt dar. ${ }^{74}$ Im Gegenteil: Um den »Triumph von hierarchischer Ordnung über (Rechts-)Sinn « 75 einzudämmen, müssten das Rechtliche und das Politische entzweit werden. Erbarmungslos und in aller Konsequenz. Diese waghalsige Demontage von Luhmanns Funktionssynthese ${ }^{76}$ ist folgenschwer und folgenreich: Sie schafft ein Problem, das Cover, ganz amerikanisch, als $\gg \mathrm{He}-$ rausforderung " kennzeichnet, das alteuropäisch aber als Tragödie apostrophiert werden kann und im heutigen Wissenschaftsjargon doublebind-Situation genannt wird: »The challenge presented by the absence of a single sobjectives interpretation is ... the need to maintain a sense of legal meaning despite the destruction of any pretense of superiority of one nomos over another. « 77 Was empfiehlt Cover in dieser Lage rechtspluralistischer Blockade?

Temporalisierung des Rechts bzw. seiner Entfaltung - so lässt sich sein Ratschlag wohl am ehesten beschreiben. Aber Temporalisierung in einem ganz bestimmten Sinn, nach einem Muster, das, wie noch zu zeigen ist, in seiner Grundstruktur von jüdisch-rechtlichem Gedankengut inspiriert wird. Um dies zu erläutern, muss näher auf Covers Konzept der interpretative commitments einer nomischen Gemeinschaft eingegangen werden. Denn diese commitments - die wir hier mit dem Begriff der interpretativen Selbstbindung (einer Gemeinschaft) übersetzen - sind, wie schon erwähnt, in Covers Vorstellung auf's Engste mit der Genese von Recht verknüpft. Die Frage lautet: Wie kann Interpretation eines Textes der Legalität Rechtssinn (als genetische Bedingung des Rechts) schaffen? Für Cover bedarf es zweierlei: (I) Zunächst muss diese Interpretation eine Selbstbindung der nomischen Gemeinschaft an eine »teleologische Vision « reflektieren. Darunter versteht Cover eine »programmatische $\mathrm{Ab}$ sichtserklärung «, mit welcher die Gemeinschaft
70 COVER (1983) 7: »The normative universe is held together by the force of interpretative commitments - some small and private, others immense and public. These commitments - of officials and of others - do determine what law means and what law shall be. If there existed two legal orders with identical legal precepts and identical, predictable patterns of public $\%$ force, they would nonetheless dif- of the orders, the precepts were universally venerated while in the other they were regarded by many as fundamentally unjust. « Der Begriff »interpretative commitments « ist deshalb glücklich gewählt, weil - wie im wiedergegebenen Zitat anklingt - die Überzeugungen, die man hat und praktiziert, die eigene Weltkonstruktion bestimmen, d. h. die Welt zu deuten, zu interpretieren erlauben.

7I Cover (I983) I I.
72 Cover (I983) $40 \mathrm{ff}$.

73 Cf. Stone (I993) 825.

74 Cover (I983) 43: »The position that only the state creates law [sc. legal meaning] thus confuses the status of interpretation with the status of political domination «.

75 Cover (1983) 58.

76 Cf. oben S. I7.

77 Cover (1983) 44. 
das ihr eigene Streben (striving) nach Verwirklichung ihrer Weltanschauung in Rechtsform zum Ausdruck bringt. (2) Alsdann muss die Interpretation von einem paideiistischen Willen getragen sein: Sie soll der Gemeinschaft erläutern und verstehen helfen, welche juristischen Obligationen von der teleologischen Vision verlangt werden, an die sie sich (selbst) gebunden hat. Sind diese zwei Voraussetzungen im Rahmen des textinterpretativen Prozesses erfüllt, ist Rechtssinn entstanden - und damit Recht, das diesen Namen unabhängig davon verdient, ob es der »offiziellen « Interpretation des Staates entspricht oder nicht. ${ }^{78}$

Erkennbar wird hier aber: Wenn interpretative Selbstbindungen Recht schaffen, kann in einem Staat (der schon längst keine Glaubensgemeinschaft mehr ist) eine Pluralität von Rechten emergieren. Denn die pluralistische Gesellschaft kennt viele (nomische) Gemeinschaften, die alle Recht generieren können. Dann wird das Problem des double-bind aktuell. Cover sieht, dass "Jurispathie « nicht zu vermeiden ist: Staatliche Gerichte werden immer ein »offizielles « Recht festlegen, ja müssen dies auch tun. ${ }^{79}$ "Jurispathie " ist ein Dauerzustand, eine perennierende Schwäche, ohne Aussicht auf Behebung. Aber und das ist der springende Punkt - der »Jurispathie « kann auch ein sozialer Mehrwert abgerungen werden, sofern man nur bereit ist, Rechtspluralismus ernst zu nehmen, d.h. jeden unter Bedingungen interpretatorischer Selbstbindung generierten Rechtssinn als »Recht « anzuerkennen. »Jurispathie « kann nämlich insofern auch virtuos (und nicht nur vizios) sein, als die Kollision der Rechte von Staat einerseits und von nomischen Gesellschaften andererseits einen evolutorischen Prozess der Verifikation der verschiedenen (kollidierenden) interpretativen Selbstbindungen in Gang setzt. Sichergestellt wird über diese Temporalisierung der Rechtsentfaltung, dass das sich durchsetzende Recht dauerhaft mit Rechtssinn bewehrt bleibt, also stets einer »teleologischen Vision" dient. Das "offizielle " Recht kann sich m.a.W. nicht mehr damit begnügen, sich als das einzig existierende »Recht « zu präsentieren, weil es vom politischen Gesetzgeber erlassen wurde. Die Referenz auf auctoritas reicht nicht mehr aus, um das »offizielle « Recht zu legitimieren. Im Coverschen Rechtsmodell baut der »Wettbewerb der Rechte « einen dauerhaften Legitimationsdruck auf das »offizielle" Recht auf. Denn: Falls die interpretative commitments, die das "offizielle « Recht fundieren, sich über die Zeit abschwächen oder auflösen sollten, oder falls es den Gerichten nicht mehr gelingen sollte, dieses Recht paideiisch verständlich zu machen, so besteht die Chance, dass ein alternatives Recht, welches irgendeine nomische Gemeinschaft geschöpft hat, über kurz oder lang die Oberhand erlangt. Das Gleiche gilt aber auch für die verschiedenen nomischen Gemeinschaften, die ihre Interpretation des Legaltextes gegen das "offizielle « Recht (im Sinne der Entwicklung von texts of resistance) immer wieder verteidigen müssen. ${ }^{8 \circ}$ In diesem evolutorischen Prozess der Resignifikation des Texts der Legalität manifestiert sich also der Grundsatz, dass sämtliche konkurrierenden Interpretationen von Staat und nomischen Gemeinschaften "Recht " im gleichen Maße, im gleichen Sinne darstellen. ${ }^{8 \mathrm{I}}$ Diese Pluralität der Interpretationen unterscheidet aber gleichzeitig Covers Ansatz von solchen kantischer Provenienz. Denn, obwohl letztere das Recht nicht auf schiere auctoritas zurückführen, behandeln sie es trotzdem als Produkt der Vernunft, was nichts anderes heißt, als dass sie im Recht ein kohärentes System von Rechtsprinzipien oder in Barthes' Worten einen Text sehen, der in sich
78 Cf. zum Ganzen Cover (1983) $44 \mathrm{ff}$.

79 Cover (I983) 40 ff., 53 ff.

80 BERMAN spricht in diesem Kontext von "jurispersuasion "; cf. BERMAN (2005) 533-538, 538: "Likewise, a non-state jurisdictional assertion must make a strong case to the governments of the world and other political actors that the assertion of community dominion is appropriate and that the sub- stantive norms expressed are worth adopting."

8I Cf. Cover (I983) 52: »Our overriding temptation in the absence of substantial, direct, and immediate violent resistance to official law is to concede the state's principal claim to interpretation and to release the jurisgenerative processes of associations, communities, and movements to a delegated, secondary, or interstitial status. For those unwilling to conceive of law in such a state-bound framework, however, the law-creating processes of the Quakers, Amish, and other groups that have made their relation to the violence of the state a central normative question assume a special significance. This significance lies in the group's creation of a jurisprudence that orders the forms and occasions of confrontation, a jurisprudence of 
einen festen und endgültigen Sinn (die Vernunft) verbirgt. ${ }^{82}$

Die Wahlverwandtschaften von Covers Bild des Rechts mit jüdischem Rechtsdenken liegen auf der Hand und werden von ihm auch offen gelegt. Das Verdienst, das volle Ausmaß der Anleihen Covers an die jüdische Rechtskultur aufgedeckt zu haben, kommt aber Suzanne Last Stone zu. ${ }^{83}$ Covers pluralistisches Rechtsmodell deutet sie als Konkretisierung dreier jüdischrechtlicher Kerngedanken:

I. Anarchisches Recht: Weil das jüdische Recht keine institutionelle Hierarchie kennt, lässt es eine Vielfalt von Interpretationen zu. »[T]he rabbis revealed in the splethora of laws They were able to do exactly what modern theory finds problematic - continue in the face of radically inconsistent and plural understanding of the law. ${ }^{84}$ Die Rabbiner haben einen Mythos der Legitimität für ein radikal diffuses Autoritätssystem kreiert. Dieser Mythos ist die talmudische Geschichte der himmlischen Stimme, die zwischen sich widersprechenden Rechtsansichten der Schulen von Hillel und Schammai mit dem Satz vermittelt hat: "Die eine und die andere Rechtsansicht sind beide die Worte des Lebenden Gottes. «In Stones Augen hat Cover diesen Mythos einer modernen Lektüre zugeführt, indem er im evolutorischen Kollisionsprozess der verschiedenen Rechtsinterpretationen einen gesellschaftlich virtuosen und sinnträchtigen Vorgang erblickt hat.

2. Paideiisches Recht: In der jüdischen Tradition ist Recht aktiv. Recht ist, was jedes Gemeinschaftsmitglied aus innerer Überzeugung tut, und nicht, was ihm vom Staat vorgegeben, oktroyiert wird, zu was es gezwungen wird. Dieser Sicht der Dinge habe Cover - so Stone einen modernen Sinn abgewonnen, um die Funktion des Gerichts nicht in der "Nötigung « von
Recht anzusiedeln, sondern darin, einer nomischen Gemeinschaft zu erläutern, wo der tiefere Sinn ihres Rechts liege. Recht sei für Cover demnach nicht Macht oder Weberianischer Zwang, sondern Paideia: "The law imposes obligations that community members obey because they understand the law as embodying their common goals and aspirations. Legal interpretation in any nomic community ... achieves meaning by drawing out ... the values and implications of the obligations imposed by the community's law. ${ }^{85}$ Darauf fuße auch, so meint Stone weiter, Covers zentrale Vorstellung der Genese von Recht durch interpretative Selbstbindung. ${ }^{86}$

3. Transformatorisches Recht: Schließlich zeigt Stone auch, wie sich Cover Elemente der Maimonidesschen Rechtsphilosophie zunutze macht. Diese verstand den Zweck der mitzvot (Gebote) der Tora als Hilfe für die Menschen in ihrem Streben nach Perfektion: "For Maimonides, the Torah is divine because it is concerned with human transformation and not solely with the mechanics of conventional governance. ${ }^{87}$ In der Tat: Cover fasst Recht keineswegs als bloßes Instrument sozialer Kontrolle auf. Vielmehr betrachtet er es als Vehikel der gesellschaftlichen Transformation, das durch die Pädagogie der Gerichte angetrieben wird. Das wird vor allem daran offenkundig, dass in Covers Lehre die (vorne umschriebene) »teleologische Vision « eine unerlässliche Komponente des Rechts darstellt. In diesem Sinne schreibt er denn auch: »By themselves the alternative worlds of our visions ... dictate no particular set of transformations or efforts of transformation. But law gives a vision depth of field, by placing one part of it in the highlight of insistent and immediate demand ... ${ }^{88}$

Was ist mit Covers Rechtsmodell gewonnen, was verloren? Der eigentliche Ertrag ist, in aller resistance that is necessarily also one of accommodation."

82 So z. B. Goebel (2003) 372:

"Aufgrund der Rekonstruktion des Rechts als ein konsistentes und kohärentes System von Grundsätzen und Regeln kann zumindest die Hoffnung gepflegt werden, dass im System der vernünftige Gemeinwille der republikanischen ๖ั้ $\quad$ scheint."
83 Stone (I993).

84 StONe (I993) 828.

85 Stone (r993) 830f.

86 STONe (I993) 83 I.

87 StOne (I993) 832.

88 Cover (I983) 9. 
Radikalität formuliert, der verdiente Tod des Gesetzgebers. Cover lässt nämlich den Verzicht auf die Abhängigkeit der Rechtsinterpretation von einer allmächtigen und allwissenden Instanz (früher: Kaiser oder Papst; heute: der Gesetzgeber) realistisch erscheinen - realistisch in dem Sinne, dass der modus operandi des Rechts von diesem Verzicht nicht beeinträchtigt wird. Das Axiom einer vorgegebenen, apodiktischen, gleichsam metaphysischen auctoritas wird entbehrlich. Die Suche nach einer einzig richtigen »Wahrheit « wird ausgetauscht gegen eine Pluralität gleichmäßig legitimer Interpretationen des Textes der Legalität. Dieser Rechtspluralismus erlangt dann die Qualität einer ahistorischen Suche nach Recht durch gleichmäßige, "gleichberechtigte "Relevanz von vergangenen, gegenwärtigen und auch zukünftigen Rechtsinterpretationen, so dass die verbindliche (»offizielle«) Interpretation des Legaltextes immer unter Revisionsvorbehalt steht.

Die Schwierigkeit, die Covers Rechtsverständnis für die westliche Rechtskultur - also für uns Kinder Max Webers - schafft, liegt in der Vorstellung einer Ubiquität des Rechts, in dieser jüdischen Auffassung, dass das (z. B. vom staatlichen Gericht) einmal determinierte, »offizielle " Recht nicht koextensiv ist mit "Recht « schlechthin. Alle Rechtsinterpretationen, die Covers Kriterien der interpretativen Selbstbindung einer nomischen Gemeinschaft genügen, sind »Recht «. Die hic et nunc erfolgende Determination von Recht hat somit keine Finalität, keine Endgültigkeit, weil multiple »normative Wahrheiten " vorstellbar sind. Deshalb geht, ganz halachisch gedacht, »offizielles « Recht immer mit der Möglichkeit der eigenen Modifikation schwanger. Dass Recht einmal determiniert ist, setzt andere Rechtsinterpretationen nicht "außer Kraft «. Aber diese Vorstellung ist nur problema- tisch, solange man sich weigert, Recht als evolutorischen Prozess zu verstehen. Dass dem so ist, dass Recht ein Fluss ist, in den man nicht zweimal steigen kann, lehrt uns die Geschichte.

Was folgt daraus für die (hier vertretene) Sicht von Recht als Lehre eines besonderen Umganges mit dem Text? Covers Rechtsverständnis erlaubt es, Barthes' "Skriptor " ein juristisches Kleid zu geben. Rechtsinterpretation wird zu einer kreativen Tätigkeit, und zwar in aller Brutalität: Der Exeget - und er allein! - verleiht dem Text seinen Sinn. Das Ziel der Interpretation ist nicht die Entdeckung der Absicht des Texturhebers - die Absicht Gottes, des Kaisers, des Papstes oder des Gesetzgebers -, sondern die Generierung von »Rechtssinn «, die aber immer an die Voraussetzung der interpretativen Selbstbindung einer nomischen Gemeinschaft gebunden ist. Nur dann hat man es mit »Recht « im pluralistischen Sinne Covers zu tun (und nicht mit einem individuell-subjektiven, rechtlich [noch] irrelevanten Vorschlag des Interpreten).

III. Die Mutation:

Transnationales Recht und Paideia

Jetzt können wir unsere These in ihrer Endfassung formulieren: In der Transnationalität wird das Recht anarchistisch, paideiistisch, transformatorisch. In diesem Drift widerspiegeln sich die "entterritorialisierten "Strukturen der Weltgesellschaft, namentlich der Umstand, dass die Produktion von Weltrecht "politikfern « vor sich geht. Diese Mutation des Rechts in Richtung Coverscher Rechtsprinzipien ist aber ein kriechender, schleppender und schwerfälliger Prozess. Keineswegs bildet sich ein Weltrecht spontan heraus. Die Mutation des Rechts im trans- 
nationalen Raum erfolgt vielmehr stufenweise, graduell, in kleinen Schritten. Und damit ist es nicht getan. Hinzukommen müssen noch Selektion und Retention der mutierten Rechtselemente im transnationalen Rechtssystem. ${ }^{89}$ Nur dann hat die Rechtsmutation auch tatsächlich die Strukturen dieses Systems verändert.

Bewegungen im Sinne dieses evolutorischen Musters lassen sich im Europäischen Privatrecht erkennen. Freilich nicht in abgeschlossener Form, aber zumindest ansatzweise - und wir deuten diese ersten Spuren Coverscher Rechtstheorie als einen generalisierbaren Trend im transnationalen Recht, der sich in Zukunft verstärken wird. In nuce:

Europäisches Privatrecht ist vor allem Richtlinienrecht. Das Konzept der europäischen Richtlinie ist ursprünglich ganz rechtshierarchisch gedacht: Nach Art. 249 Abs. 3 EGV sind Richtlinien für die Mitgliedsstaaten »hinsichtlich des $\mathrm{zu}$ erreichenden Ziels « verbindlich. Damit ist im Grunde nichts anderes als eine Normenpyramide aufgestellt. Diese Verbindlichkeit von Richtlinien ist aber (mindestens de facto) mit der Verbindlichkeit von nationalstaatlichen Gesetzen nicht vergleichbar. Dass es nämlich der EuGH für nötig gehalten hat, im Laufe der letzten zwei Jahrzehnte (nebst der doctrine of direct effects ${ }^{9}$ ) ein im EGV nicht vorgesehenes methodisches Instrument, das Gebot der richtlinienkonformen Auslegung nationalen Rechts, zu entwickeln, um die Effektivität von Richtlinien zu steigern, ist viel bezeichnender als gemeinhin angenommen. Denn diese judizielle Erfindung beruht letztlich auf der Erkenntnis, dass Rechtshierarchien sich im transnationalen Raum nicht reproduzieren lassen. Die schiere Existenz des Gebots richtlinienkonformer Interpretation zeugt von der intensiven Suche nach alternativer, eben transnationaler Normativität, und zwar, um ganz präzis zu sein, nach einem nichthierarchischen Umgang mit dem Text der Legalität. Diese reichlich verkürzten Ausführungen bedürfen der Erläuterung.

Was genau besagt das Gebot der richtlinienkonformen Auslegung? In den Urteilen Von Colson und Kamann ${ }^{91}$ sowie $\mathrm{Harz}^{92}$ hatte der EuGH zum ersten Mal eine Verpflichtung der mitgliedstaatlichen Gerichte angenommen, im Rahmen ihrer Zuständigkeiten »bei der Anwendung des nationalen Rechts, insbesondere auch der Vorschriften eines speziell zur Durchführung ... [einer] Richtlinie ... erlassenen Gesetzes, dieses nationale Recht im Lichte des Wortlauts und des Zwecks der Richtlinie auszulegen ..., um das in Artikel I 89 Abs. 3 [sc. 249 Abs. 3] genannte Ziel zu erreichen ${ }^{93}$ Diese Formulierung, die noch dahingehend präzisiert wurde, »dass eine richtlinienkonforme Auslegung nur geboten ist, wenn das einschlägige nationale Recht die Möglichkeit zu einer solchen durch das Vorhandensein eines entsprechenden >Beurteilungsspielraums« offen lässt «, ${ }^{94}$ wurde dann im Urteil Marleasing drastisch verschärft. Ausgeführt wurde darin, dass das Gebot richtlinienkonformer Auslegung die Interpretation in einer bestimmten, mit der einschlägigen Richtlinie unvereinbaren Weise » verbietet «. ${ }^{95}$ Gleichwohl bleibt der EuGH ambivalent, wenn er die Rigorosität dieser Wendung, die den Schluss nahe legt, die Richtlinienkonformität genieße Vorrang vor allen innerstaatlichen Auslegungskriterien, mit der Aussage relativiert, das nationale Gericht müsse die Interpretation innerstaatlichen Rechts »soweit wie möglich am Wortlaut und Zweck der Richtlinie ausrichten $\ll .{ }^{96}$
89 Denn die Mutation stellt sich als solche bloße Variation dar; zur Trias der evolutorischen Mechanismen Variation/Selektion/ Retention Amstutz (200I) i I I f. $90 \mathrm{EuGH}$ vom 4.I2.I974, Rs. 4I/74, Slg. I974, I337, Van Duyn; EuGH vom 5.4.I979, Rs. I48/78, Slg. I979, I629, Ratti; EuGH vom 26.2. I 986 , Rs. I 52/84, Slg. I986, 723, Marshall.

9I EuGH vom Io.4.I984, Rs. I4/83, Slg. I984, I89 I ff., Von Colson und Kamann.
$92 \mathrm{EuGH}$ vom Io.4.I984, Rs. 79/83, Slg. I984, I922 ff., Harz.

$93 \mathrm{EuGH}$ vom Io.4.I984, Rs. I4/83, Slg. I984, Randnr. 26, Von Colson und Kamann; EuGH vom I0.4.I984, Rs. 79/83, Slg. I984, Randnr. 26, Harz; cf. zum Hintergrund dieser Rechtsprechung statt anderer BRECHMANN (I994) $47 \mathrm{f}$., $50 \mathrm{f}$.

94 So die Zusammenfassung von CANARIs (2002) 60, in Anlehnung an EuGH vom Io.4.1984, Rs. I4/83, Slg. I 984 , Randnr. 28,
Von Colson und Kamann, und EuGH vom Io.4.I984, Rs. 79/83, Slg. I984, Randnr. 28, Harz. 95 EuGH vom I3.I I.I990, Rs. CI06/89, Slg. I990, Randnr. 9, Marleasing.

96 EuGH vom I3.I I.I990, Rs. CI06/89, Slg. I990, Randnr. 8, Marleasing. 
Wie muss man diese enigmatischen Formulierungen verstehen? In der Lehre ist die Frage ungelöst. Von den einen wird die MarleasingRechtsprechung dahingehend aufgefasst, dass Gemeinschaftsrecht mitsamt seinen Richtlinien nationalem Recht stets vorgehe, weshalb die richtlinienkonforme Auslegung Vorrang vor den nationalen Auslegungsmethoden genieße. Die anderen versuchen, die Vertikalität bruchlos ineinander fließender völker- und nationalrechtlicher Hierarchien durch den Einbau von »Abwägungsoperationen « zu retten: Der EuGH gebiete den nationalen Gerichten nur, die Frage zu prüfen, ob das nationale Recht mehrere Auslegungsmöglichkeiten zulasse, und ggf. unter diesen diejenige vorzuziehen, die zu einem richtlinienkonformen Ergebnis führe. Beiden doktrinären Ansichten ist indes gemein, nach Wegen zu suchen, um die mit der Entscheidung Marleasing verloren gegangene Hierarchie des Rechts zu restaurieren, sei es auf gemeinschaftsrechtlicher, sei es auf nationalstaatlicher Ebene. ${ }^{97}$

Beide Wege führen in die Irre. Weiter kommt man nur, wenn man die Marleasing-Entscheidung als fundamentale Enthierarchisierung des Rechts im transnationalen Raum versteht. Und man sollte sogar noch einen Schritt weiter gehen, will man die ganze Tragweite dieser Entscheidung erschließen. Man sollte nämlich zur Kenntnis nehmen, dass mit dieser Rechtsprechung anarchisierende, paideiisierende und transformatorische Ströme in das Meer des Europäischen Privatrechts eingeflossen sind. Ströme, die unverkennbar mit Ingredienzien der Rechtstheorie Covers durchzogen sind:

I. Dem Grundkonzept der Richtlinie entsprechend lässt das Urteil Marleasing Rechtspluralismus in der Gemeinschaft zu. Vor dem Hintergrund der vorliegend entwickelten Gedanken bedeutet das: Den Mitgliedstaaten wird im Eu- ropäischen Privatrecht eine Coversche »Anarchie« im Anwendungsbereich der betreffenden Richtlinie gestattet. Nun ließe sich einwenden, dass dieser Rechtspluralismus in Wahrheit kein solcher sei, weil er ja nur hingenommen werde, sofern das Richtlinienziel gewährleistet sei. Aber hier sollte man die Sprache des EuGH in der Marleasing-Entscheidung ganz genau betrachten: Worauf will das Gericht mit der Wendung hinaus, das nationale Gericht müsse die Interpretation innerstaatlichen Rechts "soweit wie möglich " am Wortlaut und Zweck der Richtlinie ausrichten? Welcher Sinn lässt sich diesem "soweit wie möglich « abgewinnen?

Damit werden, so meinen wir, die virtuosen Effekte der Coverschen Temporalisierung des Rechts im Europäischen Privatrecht ausgebeutet. In dem Sinne nämlich, dass in der wiedergegebenen Passage - anders als in Art. 249 Abs. 3 EGV - nicht nur die rechtliche Freiheit der Mitgliedstaaten, über die Form und die Mittel der Umsetzung zu befinden, sondern auch deren faktische Freiheit, über die Umsetzungsvornahme als solche zu entscheiden, ausdrücklich in Rechnung gestellt wird. Dadurch werden Chancen geschaffen, Chancen, dass sich das Coversche Temporalisierungsmuster im transnationalen Raum des Europäischen Privatrechts entfalten kann: Durch die stets möglich bleibende Kollision voneinander abweichender nationaler »Privatrechte « - denn die umgesetzten und nichtumgesetzten nationalen Rechte werden in der Marleasing-Rechtsprechung alle als »Recht « im Coverschen Sinne betrachtet - wird die evolutorische Spannungslage erzeugt, die Cover zufolge erforderlich ist, um auf Dauer sicherzustellen, dass immer nur Recht mit »Rechtssinn « determiniert wird. Mit der Hervorhebung der umfassenden Freiheit der Mitgliedstaaten zur Richtlinienumsetzung wird letztlich signalisiert,

97 Cf. zum Ganzen eingehend AMSTUTZ (2003b) m. Nw. 
dass der EuGH die Mitgliedstaaten als nomische Gemeinschaften auffasst, die selber über ihre interpretatorischen Selbstbindungen (im Sinne Covers) entscheiden. Dass sich die Mitgliedstaaten im Falle der Nichtumsetzung Vollstreckungsmaßnahmen und haftungsrechtlichen Risiken (auch nach der Frankovich-Rechtsprechung) aussetzen, ändert an diesem Befund nichts, ja entspricht letztlich nur der von Cover unterstrichenen Tatsache, dass "Jurispathie « eben nie und nimmer zum Verschwinden gebracht werden kann.

2. Die Marleasing-Rechtsprechung besitzt sodann auch eine paideiistische Dimension, die man nicht unterschätzen sollte. Das folgt zwar schon aus dem Umstand, dass der EuGH nicht die Anwendung des nationalen Privatrechts, sondern die Auslegung des Richtlinienprivatrechts zur Aufgabe hat (Art. 220 Abs. I EGV). Aber auch hier ist die im Urteil Marleasing benutzte Wendung des "soweit wie möglich « von besonderer Bedeutung. Mit deren Ambivalenz geht einher, dass der EuGH nicht auf bloßen Zwang, sondern eben auch auf pädagogische Aufklärung setzt: Indem semantisch die Möglichkeit einer fehlerhaften Umsetzung einberechnet wird, erhält die Begründung des Urteils einen ganz anderen Stellenwert als in nationalstaatlichen Gerichtsentscheidungen. Während in letzteren die Urteilsgründe eine klar rechtfertigende Ausrichtung haben - Cover würde sagen: Rechtfertigung des Einsatzes von (Staats-)Gewalt -, eignet ihnen in Urteilen des Vorabentscheidungsverfahrens (Art. 234 EGV) eine andere Qualität an: Hier geht es darum, den mitgliedstaatlichen Gerichten zu erläutern, wie eine Richtlinienbestimmung zu verstehen ist, damit diese Gerichte in die Lage versetzt werden, das nationale Privatrecht entsprechend anzuwenden. Diese Frage kann der EuGH unmöglich - schon mangels Fachkenntnissen im einschlägigen nationalen Recht - selbst beantworten. Vielmehr vermögen seine Entscheidungsmotive allein eine Erklärungsfunktion auszuüben, die darauf hinzielt, die Operationen des betroffenen mitgliedstaatlichen Rechtssystems im Sinne der Richtlinie zu beeinflussen.

3. Das Marleasing-Recht ist schließlich auch transformatorisch im Sinne Covers: Sein unmittelbares Anliegen ist nicht in der sozialen Kontrolle zu sehen, ${ }^{98}$ sondern vielmehr, um mit Weiler zu sprechen, in der »transformation of Europe «. ${ }^{99}$ Was auf dem Spiel steht, ist Arbeit an der europäischen Gesellschaft als solcher, konkret: die Herstellung dynamischer Vernetzungen von europäischen Zivilrechtsordnungen, dies mit dem Ziel, in diesen Ordnungen »normative Kompatibilitäten « zu schaffen. ${ }^{\text {Io० }}$ Man kann hier die gewichtigen Formeln von Wiethölter verwenden: In der Marleasing-Rechtsprechung geht es um »Schleusen-Wartungen «, d.h. um juristische Arbeit »an den >Grenzen`von System und Umwelt ..., an denen über sstrukturelle Kopplungen ‘ oder `Externalisierung ‘ verhandelt und entschieden wird ", um Rechtsarbeit, "an den `Passagen $<. .$. , in denen Geltung und Anerkennung durchzusetzen sind $« .{ }^{\text {Ior }}$ Vielleicht liegt in diesem transformatorischen Zug - das wäre unsere Arbeitshypothese für die Zukunft - die bislang allzu wenig berücksichtigte Haupteigenschaft des transnationalen Rechts.

Marc Amstutz, Vaios Karavas

98 So auch Teubner (I996b) 27I.

99 WeILER (I99I).

ıoo Cf. dazu eingehend Amstutz

(2005).

IOI WiETHÖLTER (2003) I $8 \mathrm{f}$. 
Literatur

Agamben, Giorgio (2002), Homo Sacer. Die Souveräne Macht und das nackte Leben, Frankfurt: Suhrkamp.

Amstutz, Marc (200I), Evolutorisches Wirtschaftsrecht. Vorstudien zum Recht und seiner Methode in den Diskurskollisionen der Marktgesellschaft, Baden-Baden: Nomos.

- (2003a), Das Gesetz, in: K(l)eine Festschrift für Pierre Tercier, hg. von P. Gauch, P. Pichonnaz, Zürich: Schulthess, I 55-I65.

- (2003b) Zwischenwelten. Zur Emergenz einer interlegalen Rechtsmethodik im europäischen Privatrecht, in: Rechtsverfassungsrecht. Recht-Fertigung zwischen Privatrechtsdogmatik und Gesellschaftstheorie, hg. von C. Joerges, G. Teubner, BadenBaden: Nomos, 2I3-237.

- (2005), Normative Kompatibilitäten. Zum Begriff der Eurokompatibilität und seiner Funktion im Schweizer Privatrecht, in: Schweizerisches Jahrbuch für Europarecht $(2004 / 5)$, hg. v. A. EPINEY u. a., 235-25I.

BARTHES, Roland (I986), Die Lust am Text, Frankfurt: Suhrkamp.

- (2000), Der Tod des Autors, in: Texte zur Theorie der Autorschaft, hg. von F. Jannidis, G. LaUer, M. Martinez, S. Winko, Stuttgart: Philipp Reclam jun., I 85I93.

BECK, UlRICH (I997), Was ist Globalisierung? Irrtümer des Globalismus - Antworten auf Globalisierung, Frankfurt: Suhrkamp.

Berman, Paul Schiff (2002), The Globalization of Jurisdiction, in: University of Pennsylvania Law Review I 5 I, 3 I I-529.

- (2005), From International Law to Law and Globalization, in: Columbia Journal of Transnational Law 43, 485-556.

BrechmanN, WinfRIED (I994), Die richtlinienkonforme Auslegung. Zugleich ein Beitrag zur Dogmatik der EG-Richtlinie, München: Beck.

Burton, John W. (I972), World Society, Cambridge: Cambridge University Press.

Calliess, Gralf-Peter (200i), Globale Kommunikation - staatenloses Recht. Zur (Selbst-) Regulierung des Internet durch prozedurales Recht, in: Globalisierung als Problem von Gerechtigkeit und Steuerungsfreiheit des Rechts, hg. von M. ANDERHEIDEN, S. Huster, S. Kirsten, 6I-79.

- (2002), Reflexive Transnational Law. The Privatisation of Civil Law and the Civilisation of Private Law, in: Zeitschrift für Rechtssoziologie, I85-21 6 .

Canaris, Claus-Wilhelm (2002), Die richtlinienkonforme Auslegung und Rechtsfortbildung im System der juristischen Methodenlehre, in: Im Dienste der Gerechtigkeit: Festschrift für Franz Bydlinski, hg. von H. Koziol, P. Rummel, Wien: Springer, 47-IO3.

Cover, Robert M. (I983), The Supreme Court I982 Term Foreword: Nomos and Narrative, in: Harvard Law Review 97, 4-68.

- (I987), Obligation: A Jewish Jurisprudence of the Social Order, in: Journal of Law and Religion 5, 65-74.

Eco, Umberto (I998), Das offene Kunstwerk, 8. Aufl., Frankfurt: Suhrkamp.

ENGISCH, KARL (I935), Die Einheit der Rechtsordnung, Heidelberg: Carl Winter.

EWald, William (I995), Comparative Jurisprudence (II). The Logic of Legal Transplants, in: American Journal of Comparative Law 43, 489-5 IO.

Fischer-Lescano, Andreas u. ChrisTensen, Ralph (2005), Auctoritatis interpositio. Die Dekonstruktion des Dezisionismus durch die Systemtheorie, in: Der Staat 44, 2I3-24I.

Foucault, Michel (2000), Was ist ein Autor?, in: Texte zur Theorie der Autorschaft, hg. von F. JANNIDIs, G. Lauer, M. Martinez, S. WinKo, Stuttgart: Philipp Reclam jun., I98-229.

Giddens, Anthony (I995), Konsequenzen der Moderne, Frankfurt: Suhrkamp.

Goebel, JoACHIM (2003), Das System privatrechtlicher Wertung und die demokratische Genese des Rechts, Überlegungen zum internen $\mathrm{Zu}$ sammenhang der Privatrechtsordnung mit der Idee der kantischen
Republik, in: Archiv für Rechtsund Sozialphilosophie 89, 372386.

Habermas, Jürgen (I998), Die postnationale Konstellation. Politische Essays, Frankfurt: Suhrkamp.

Hayek, Friedrich A. von (I973), Law, Legislation and Liberty. A New Statement of the Liberal Principles of Justice and Political Economy, Volume I: Rules and Order, Chicago, London: The University of Chicago Press.

Höffe, Otfried (I999), Demokratie im Zeitalter der Globalisierung, München: Beck.

Joerges, Christian u. Neyer, Jürgen ( I997), From Intergovernmental Bargaining to Deliberative Political Processes. The Constitutionalisation of Comitology, in: European Law Journal 3, 273-299.

Kennedy, Duncan (I989), Comment on Rudolf Wiethölter's » Materialization and Proceduralization in Modern Law « and »Proceduralization of the Category of Law», in: Critical Legal Thought. An American-German Debate, hg. von C. Joerges, D. Trubek, Baden-Baden: Nomos, 5 I I-524.

Kelsen, Hans (I960), Reine Rechtslehre. Das Problem der Gerechtigkeit, 2. Aufl., Wien: F. Deuticke.

Kiesow, RaIner Maria (2004): Weltrecht Ruinenrecht, in: Kursbuch I 5 5, 98-IO7.

Ladeur, Karl-Heinz (I999), The Theory of Autopoiesis as an Approach to a better Understanding of Postmodern Law - From the Hierarchy of Norms to the Heterarchy of Changing Patterns of Legal Inter-relationships, in: EUI LAW Paper I999/03.

- (2004), Methodology and European Law - Can Methodology Change so as to Cope with the Multiplicity of the Law?, in: Epistemology and Methodology of Comparative Law, hg. von M. v. Hoecke, Oxford: Hart, 9I-I2I.

LANGEWIESCHE, DiETER (2000), Nation, Nationalismus, Nationalstaat in Deutschland und Europa, München: Beck.

Legendre, Pierre (I989), »Die Juden interpretieren verrückt «. Gutachten zu einem klassischen Text, in: 
Psyche. Zeitschrift für Psychoanalyse und ihre Anwendungen 43, 20-39.

- (200I), Die Bresche. Bemerkungen zur institutionellen Dimension der Shoah, in: Tumult. Schriften zur Verkehrswissenschaft 26, 25-32.

- (2004), Die Fabrikation des abendländischen Menschen, Wien: Turia + Kant.

Legrand, Pierre (I997), The Impossibility of 'Legal Transplants‘, in: Maastricht Journal of Comparative Law 4, III-I24.

LuhmanN, NikLas (I97I), Die Weltgesellschaft, in: Archiv für Rechtsund Sozialphilosophie 57, I-35.

- (I98I), Ausdifferenzierung des Rechts. Beiträge zur Rechtssoziologie und Rechtstheorie, Frankfurt: Suhrkamp.

- (I993), Das Recht der Gesellschaft, Frankfurt: Suhrkamp.

- (2002), Einführung in die Systemtheorie, Heidelberg: Carl-AuerSysteme.

Mertens, Hans-Joachim (I997), Lex Mercatoria: A Self-Applying System Beyond National Law?, in: Global Law without a State, hg. von G. Teubner, Aldershot: Dartmouth, $3 \mathrm{I}-43$.

Michaels, RAlF (im Erscheinen 2006): The Re-State-ment of of NonState-Law: The State, Choice of Law and the Challenge from Legal Pluralism, Wayne Law Review, elektronisch verfügbar unter: http://papers.ssrn.com/sol $3 /$ papers.cfm?abstract_id $=809244$. (einges. am 22.I.2006).

Nafziger, James R. (1996), International Sports Law as a Process of resolving Disputes, in: International and Comparative Law Quarterly 45, I30-I49.

Nickel, Rainer (2005), Participatory Transnational Governance, in: EUI LAW Paper 2005/20.

Niggli, Marcel Alexander (2000), Zurück zu den Io Geboten? Gesetzesflut und Strafrecht, in: Aufbruch ins dritte Jahrtausend. Milleniums-Vorträge an der Universität Freiburg, hg. von A. HoLDEREGGER, Freiburg i. Ue.: Universitätsverlag, I $36-\mathrm{I} 53$.

Post, David G. (I995), Anarchy, State and the Internet. An Essay on Law-Making in Cyberspace, Journal Online Law Art. 3, elektronisch verfügbar unter: http://
www.cli.org/DPost/Xo023 ANARCHY.html (einges. am 22.I.2006).

Rafi, ANUSHeH (2004), Kriterien für ein gutes Urteil, Berlin: Duncker und Humblot.

Schmitt, CARL (I967), Die Tyrannei der Werte. Überlegungen eines Juristen zur Wert-Philosophie, in: Säkularisation und Utopie, Ebracher Studien. Ernst Forsthoff zum 65. Geburtstag, Stuttgart: Kohlhammer, 37-62.

Schütz, Anton (1997), The Twilight of the Global Polis. On Losing Paradigms, Environing Systems and Observing World Society, in: Global Law Without a State, hg. von G. Teubner, Aldershot: Dartmouth, 257-293.

Simon, DieTER (I975), Die Unabhängigkeit des Richters, Darmstadt: Wissenschaftliche Buchgesellschaft.

Simon, Thomas (2005), Geltung. Der Weg von der Gewohnheit zur Positivität des Rechts, Rechtsgeschichte 7, I00-I37.

Stein, Ursula (I995), Lex Mercatoria. Realität und Theorie, Frankfurt: Klostermann.

Stichweh, Rudolf (200I), Die Weltgesellschaft. Soziologische Analysen, Frankfurt: Suhrkamp.

- (2004), Weltgesellschaft, in: Historisches Wörterbuch der Philosophie, Bd. I2, Basel: Schwabe \& Co., 486-490.

Stone, Suzanne Last (1993), In Pursuit of the Counter-Text. The Turn to the Jewish Legal Model in Contemporary American Legal Theory, in: Harvard Law Review I06, 8I $3-894$.

Teubner, Gunther (I996a), Des Königs viele Leiber. Die Selbstdekonstruktion der Hierarchie des Rechts, in: Soziale Systeme 2, 229-255.

- (1996b), Globale Bukowina. Zur Emergenz eines transnationalen Rechtspluralismus, in: Rechtshistorisches Journal I 5, 255-290.

- (Hg., I997), Global Law Without a State, Aldershot: Dartmouth.

- (I998), Die unmögliche Wirklichkeit der lex mercatoria. Eine systemtheoretische Kritik der théorie ludique du droit, in: Festschrift für Wolfgang Zöllner zum 70. Geburtstag, hg. von M. LIEB, Köln: Heymann, 565-588.
- (2003), Der Umgang mit Rechtsparadoxien. Derrida, Luhmann, Wiethölter, in: Rechtsverfassungsrecht, Recht-Fertigung zwischen Privatrechtsdogmatik und Sozialtheorie, hg. von C. Joerges, G. Teubner, Baden-Baden: Nomos, $25-45$.

Teubner, Gunther u. Karavas, Vaios (2003), http://www.Company NameSucks.com. Drittwirkung der Grundrechte gegenüber >Privaten $<$ im autonomen Recht des Internet, in: Innovationsoffene Regulierung des Internet, hg. von K.-H. Ladeur, Baden-Baden: Nomos, 249-272.

Vesting, Thomas (2004), Die Staatsrechtslehre und die Veränderung ihres Gegenstandes. Konsequenzen von Europäisierung und Internationalisierung, in: Veröffentlichungen der Vereinigung der Deutschen Staatsrechtslehrer 63, 4I-70.

Volodine, ANToINe (I985), Biographie comparée de Jorian Murgrave, Paris: Denoël.

Watson, Alan (1993), Legal Transplants. An Approach to Comparative Law, Athens: University of Georgia Press.

- (200I), The Evolution of Western Private Law, Baltimore, London: Johns Hopkins University Press.

Weiler, Joseph H. H. (I99I), The Transformation of Europe, in: Yale Law Journal Ioo, 24032482.

WERBLOWSKY, R. J. ZWI (I962), Joseph Karo. Lawyer and Mystic, London: Oxford University Press.

Wiethölter, RUdolf (2003), RechtFertigungen eines GesellschaftsRechts, in: Rechtsverfassungsrecht, Recht-Fertigung zwischen Privatrechtsdogmatik und Gesellschaftstheorie, hg. von G. TeUBNER, C. JOERGES, Baden-Baden: Nomos, I3-2I.

WobBe, THERESA (2000), Weltgesellschaft, Bielefeld: Transcript.

Zumbansen, Peer (2002), The Privatization of Company Law? Corporate Governance Codes and Commercial Self Regulation, in: Juridicum, I36-I 45 .

- (2003), Zum Geltungsanspruch transnationalen Rechts, in: Rabels Zeitschrift für ausländisches und internationales Privatrecht 67 , 637-682. 